\title{
Kommunikation kommunaler Verwaltungsbehörden bei der Öffentlichkeitsbeteiligung: Eine qualitative Fallstudie
}

\author{
Dimitrij Umansky
}

\section{$1 \quad$ Einleitung}

Die Öffentlichkeitsbeteiligung (nachfolgend Beteiligung), verstanden als eine Teilung von Entscheidungsmacht (Arnstein 1969), stellt aus rechtlicher, normativer und empirischer Perspektive ein wichtiges Thema für Verwaltungsbehörden dar. Nach europäischem Recht sind Behörden zu einer Mindestbeteiligung verpflichtet (Müller 2010). Aus normativer Sicht sollen Behörden mit Hilfe von Beteiligung die Demokratie stärken, die Qualität ihrer Leistungen erhöhen und ihre Beziehung zur Öffentlichkeit verbessern (Canel und Luoma-aho 2018, S. 179 ff.). Auch empirische Befunde zeigen, dass eine frühe Öffentlichkeitsbeteiligung für Verwaltungsbehörden, zum Beispiel bei großen Infrastrukturprojekten, einen hohen Stellenwert einnimmt (Rademacher und Lintemeier 2015, S. 10).

Beteiligung hat insbesondere für die Kommunikation von Behörden aus verfassungsrechtlicher (Schulz et al. 2013, S. 19 ff.) und normativer Perspektive (Fredriksson und Pallas 2016, S. 159) eine wichtige Bedeutung. Aus empirischer Perspektive ist es jedoch nicht eindeutig, welche Rolle Beteiligung in der Kommunikationspraxis von Behörden tatsächlich einnimmt. Bisherige Studien zur Kommunikation von Behörden behandeln Beteiligung eher am Rande und

Mitarbeit von Jonas Bisschop.

D. Umansky $(\bowtie)$

Institut für Kommunikationsmanagement, Hochschule Osnabrück, Campus Lingen,

Lingen (Ems), Deutschland

E-Mail: d.umansky@hs-osnabrueck.de

(C) Der/die Autor(en) 2020 
kommen zu widersprüchlichen Ergebnissen (Baumgartner 2010, S. 206; Drews 2018, S. 197; Heinze 2012, S. 118). Vor allem kommunale Behörden bleiben bei dieser Frage, abgesehen von wenigen Ausnahmen (Glenn 2014), wenig erforscht. Außerdem besteht Unklarheit, inwiefern sich Behörden bei der Beteiligung um eine positive öffentliche Wahrnehmung bemühen. Es ist zwar bekannt, dass Behörden hierbei grundsätzlich eine positive Wahrnehmung fördern (Wæraas und Byrkjeflot 2012, S. 187). In Bezug auf Beteiligung ist die Außendarstellung der Behörden jedoch bisher, abgesehen von wenigen Ausnahmen (Simmons 2014, S. 316), kaum behandelt worden.

In einigen Fällen kann Beteiligung zu einer negativen öffentlichen Wahrnehmung führen, wenn sie beispielsweise die Erwartungen der Öffentlichkeit enttäuscht. Dieser Fall ist nicht unwahrscheinlich, da in der Öffentlichkeit vielfältige, teilweise gegensätzliche, Erwartungen geäußert werden können (Pfetsch 1998, S. 235). Es kann somit zu Konflikten zwischen den Beteiligungs- und Wahrnehmungszielen bei der Kommunikation von Behörden kommen (Fredriksson und Pallas 2016, S. 162). Diese Konflikte sowie ihre Auswirkungen auf die Kommunikationspraxis sind bisher jedoch kaum erforscht worden.

In diesem Beitrag wird vor dem Hintergrund der Forschungslücken eine qualitative Studie vorgestellt, die den Fragen nachgeht, 1) welche Beteiligungsziele kommunale Behörden bei ihrer Kommunikation mit der Öffentlichkeit verfolgen; 2) welche Wahrnehmungsziele die Behörden bei der Beteiligung verfolgen; sowie 3) welche Konflikte zwischen Beteiligungs- und Wahrnehmungszielen auftreten und wie sich diese auf die Kommunikation auswirken. Die Forschungsergebnisse basieren auf einer Fallstudie zur Kommunikation kommunaler Behörden beim Stromübertragungsnetzausbau. Es wurden 17 leitfadengestützte Expertengespräche mit 18 Mitarbeitern kommunaler Behörden auf Regierungs- und Verwaltungsebene geführt.

Die Ergebnisse der Studie helfen Forschenden und Praktikern, zu reflektieren, welche Rolle Beteiligung in der Kommunikationspraxis von Behörden einnimmt, und wie sich diese Rolle analysieren lässt. In den folgenden Kapiteln werden der Kommunikations- sowie der Beteiligungsbegriff im Kontext von Verwaltungsbehörden konzeptualisiert, die Relevanz der Fragestellungen herausgearbeitet, der Fall und das Forschungsdesign vorgestellt, sowie die Forschungsergebnisse dargelegt und abschließend bewertet. 


\section{Kommunikation von Verwaltungsbehörden}

Die Kommunikation von Verwaltungsbehörden wird in diesem Beitrag als strategische Kommunikation von Verwaltungen aufgefasst (Fredriksson und Pallas 2016). Sie wird definiert als Praxis zielgerichteter Teilnahme von Verwaltungsbehördenvertretern ${ }^{1}$ an einer Sinnbildung. Diese Definition basiert auf einem akteurszentrierten Verständnis von Verwaltungskommunikation (Czerwick 1998) aus der Perspektive strategischer Kommunikation (Holtzhausen und Zerfass 2013).

Die Verwaltungsbehörde als organisationaler Akteur ist der Ausgangspunkt für das Verständnis der Kommunikation. Die Kommunikation von Verwaltungsbehörden wird synonym mit dem Begriff der Verwaltungskommunikation verwendet und umfasst ,alle Kommunikationsleistungen, die im Verantwortungsbereich der öffentlichen Verwaltung erfolgen“ (Czerwick 1998, S. 489). Diese Definition entspricht den Konzepten der behördlichen Kommunikation (Drews 2018, S. 29) oder auch der public sector communication (Canel und Luoma-aho 2018, S. 33). Verwaltungsbehörden sind öffentliche Behörden und zeichnen sich u. a. durch eine Gemeinwohlorientierung aus (Canel und Luoma-aho 2018, S. 29). Unter der großen Vielfalt an öffentlichen Behörden gehören Verwaltungsbehörden entlang der horizontalen Gewaltenteilung der Exekutive an und befinden sich bei der vertikalen Gewaltenteilung auf den Bundes-, Landes- und Kommunalebenen (Bogumil 2008).

Trotz üblicher Praxis wird Verwaltungskommunikation in diesem Beitrag nicht von Regierungskommunikation differenziert. Die gängige Differenzierung beider Begriffe erfolgt aus einer normativen Unterscheidung zwischen Verwaltung und Regierung, wonach Regierungstätigkeiten eher politisch und Verwaltungstätigkeiten eher sach- und regelorientiert sind und weniger Handlungsspielraum beinhalten (Donges und Jarren 2017, S. 126). Die Unterscheidung zwischen Verwaltung und Regierung führt zu einer üblichen Differenzierung zwischen Verwaltungs- und Regierungskommunikation:

„Im Gegensatz zur politisch motivierten Regierungskommunikation leitet sich die Verwaltungskommunikation primär aus den Sachaufgaben ab, die die Behörden zu erfüllen haben." (Czerwick 1998, S. 493)

Aus empirischer Perspektive ist eine Unterscheidung zwischen Verwaltung und Regierung jedoch schwierig aufgrund ,der engen Verknüpfung zwischen der

\footnotetext{
${ }^{1}$ Als Behördenvertreter wird eine Person bezeichnet, die bei einer Behörde arbeitet.
} 
Regierung und der Verwaltung in der Praxis“ (Baumgartner 2010, S. 63). ${ }^{2}$ Die Verknüpfung wirkt sich auch auf die Kommunikationsebene aus, sodass Verwaltungskommunikation auch politische Ziele verfolgt (Franz 2013, S. 495). Zum Beispiel beeinflusst jede Information von Verwaltungsmitarbeitern und jeder Kontakt zu Bürgern die Wahrnehmung der gesamten Behörde und kann zu politischen Handlungen von Bürgern führen. Es ist davon auszugehen, dass Verwaltungsmitarbeiter vor allem in kleineren Behörden im engen Kontakt zu Regierenden stehen und stets vor dem Hintergrund politischer Leitlinien kommunizieren. Aus diesen Gründen wird im vorliegenden Beitrag nicht zwischen Verwaltungs- und Regierungskommunikation differenziert.

Verwaltungskommunikation wird hier als eine Form strategischer Kommunikation verstanden. Das Konzept der strategischen Kommunikation umfasst als Überbegriff zahlreiche Kommunikationsdisziplinen wie Public Relations, Marketing und Organisationskommunikation (Hallahan et al. 2007, S. 5 ff.). Eine gängige Definition strategischer Kommunikation lautet: „Strategic communication is the practice of deliberate and purposive communication a communication agent enacts in the public sphere on behalf of a communicative entity to reach set goals“ (Holtzhausen und Zerfass 2013, S. 74). Der organisationale Forschungsfokus strategischer Kommunikation ist zu unterscheiden von einem eher demokratie- und gesellschaftstheoretischen Fokus des Forschungsfeldes politischer Kommunikation, das die Beziehung zwischen Regierung, Medien und Bürgern thematisiert (siehe Raupp und Kocks 2018, S. 7 für Regierungskommunikation). ${ }^{3}$

Aus der Perspektive strategischer Kommunikation wird Verwaltungskommunikation als kommunikative Praxis auf individueller, organisationaler und gesellschaftlicher Ebene konstituiert (Hallahan et al. 2007, S. 14 ff.). Aus dieser praxistheoretischen Perspektive lässt sich Verwaltungskommunikation weder ausschließlich durch Gesetze auf gesellschaftlicher Ebene, Dienstvorschriften auf organisationaler Ebene noch ausschließlich durch Handlungen von Behördenmitarbeitern auf individueller Ebene erklären. Im Gegenteil bedarf es eines Zugangs zu allen drei Ebenen, um Verwaltungskommunikation zu verstehen. Aufgrund dieser

\footnotetext{
${ }^{2}$ Z. B. entscheiden zwar Regierende über Gesetzesvorschläge und ihre Umsetzung, Verwaltende können jedoch mit ihrem Fachwissen und ihrer Umsetzungserfahrung maßgeblichen Einfluss auf die Vorbereitung und Anpassung politischer Entscheidungen nehmen.

${ }^{3}$ Trotz unterschiedlicher Ausgangspunkte gibt es Überschneidungen zwischen den Feldern strategischer und politischer Kommunikation, da beide den Anspruch erheben, Makro-, Meso- und Mikro-Ebenen der sozialen Welt zu untersuchen.
} 
Vielzahl an dynamischen Einflussfaktoren, ist Verwaltungskommunikation kontextabhängig und es lässt sich daher nicht verallgemeinern, wie sie konstituiert ist.

Verwaltungskommunikation basiert als eine Form strategischer Kommunikation auf bewussten Zielorientierungen (Hallahan et al. 2007, S. 7). Eine zielorientierte Handlung steht im Widerspruch zu unreflektiertem Verhalten. Damit umfasst der vorliegende Verwaltungskommunikationsbegriff nur die Kommunikation von Behördenvertretern, die übergeordnete Ziele verfolgt. Die Zielorientierung der Kommunikation ist zum Teil von vornherein festgesetzt und entsteht zum anderen Teil im Kommunikationsverlauf (Raupp und Hoffjann 2012). Aus diesem Grund lassen sich Kommunikationsziele nicht nur aus Regeln und Normen ableiten, sondern auch aus dem Alltag heraus. Wegen der teilweise emergenten Entstehung von Kommunikationszielen beeinflussen nicht nur Behördenvertreter auf höheren hierarchischen Ebenen oder spezielle Behördenvertreter, wie Mitarbeiter der Öffentlichkeitsarbeit, die Kommunikationsziele. Auch Behördenvertreter auf unteren hierarchischen Ebenen, die nicht primär für die Kommunikation zuständig sind, gestalten Kommunikationsziele mit, während sie kommunizieren (Hallahan et al. 2007, S. 13). Entsprechend kommen alle Mitarbeiter einer Organisation als strategische Kommunikatoren in Frage, solange sie im Namen der Organisation kommunizieren. Dies steht im Widerspruch zu einem Verständnis von Regierungs-PR oder staatlicher Öffentlichkeitsarbeit, wonach nur Pressesprecher und Mitarbeiter der Öffentlichkeitsarbeit für die Kommunikation verantwortlich sind (Raupp und Kocks 2018, S. 11).

Der Kommunikationsbegriff selbst wird als Teilnahme an einer Sinnbildung verstanden (Hallahan et al. 2007, S. 23 f.). Sinnbildung wird gleichgesetzt mit der Vermittlung von Bedeutungen und Verständnissen und geschieht im Prozess einer sozialen Interaktion zwischen aktiven Teilnehmenden (Mead 1934). Somit kann Sinnbildung nicht ausschließlich von einer Seite beeinflusst werden, sondern erfolgt in einem Wechselprozess zwischen Kommunizierenden.

\section{3 Öffentlichkeitsbeteiligung}

Öffentlichkeitsbeteiligung wird vorliegend aus politischer Perspektive definiert als Machteilung, bei der die Öffentlichkeit an einer Entscheidungsmacht beteiligt wird (Arnstein 1969). Zu der Öffentlichkeit gehören ,alle vor Ort lebenden, betroffenen 
oder interessierten Menschen" (Alcántara et al. 2016, S. 15). ${ }^{4}$ Die Beteiligung an Entscheidungen lässt sich unterscheiden zwischen geringer und hoher Beteiligung (Arnstein 1969). Die Unterscheidung lässt sich stark vereinfacht in drei aufeinander aufbauenden Stufen vornehmen: Auf der untersten Stufe (1) wird die Öffentlichkeit über Sachverhalte im Zusammenhang mit Entscheidungen sowie über Entscheidungsprozesse informiert. Auf der mittleren Stufe (2) findet ein Austausch zwischen der Öffentlichkeit und den Entscheidern vor der Entscheidung statt. Vertreter der Öffentlichkeit teilen ihre Sichtweise den Entscheidern mit und es kann zu Erwiderungen beider Seiten kommen. Auf der höchsten Stufe (3) der Bürgerbeteiligung nimmt die Öffentlichkeit einen direkten Einfluss auf die Entscheidung. Der Einfluss kann sowohl formell, also gesetzlich begründet, als auch informell, zum Beispiel durch politischen Druck, erfolgen. Der Einfluss kann kooperativ, also in gemeinsamer Übereinkunft, oder konfrontativ erfolgen. Außerdem lässt sich der direkte Einfluss in Intensitätsgrade unterteilen. Im Rahmen einer bindenden Abstimmung bekommt die Öffentlichkeit die komplette Macht. Als Teil eines Gremiums teilt sich die Öffentlichkeit die Macht mit ursprünglichen Entscheidern. Insgesamt bauen die Stufen aufeinander auf und stehen in Beziehung zueinander. ${ }^{5}$

Politische Öffentlichkeitsbeteiligung ist nicht zu verwechseln mit Kommunikation (Carpentier 2016, S. 71 ff.). Neben der politischen Definition von Beteiligung als politischer Prozess (Arnstein 1969) bestehen auch kommunikationswissenschaftliche Definitionen von Beteiligung als Informationsfluss (Rowe und Frewer 2005). Das Problem einer Vermischung dieser Definitionen ist die Gleichsetzung kommunikativer Prozesse mit politischen Prozessen. So kann z. B. das Ziel eines Entscheiders, einen beidseitigen Informationsfluss zu fördern mit dem Ziel eines politischen Austausches gleichgesetzt werden, obwohl hinter dem Informationsfluss keine Absicht besteht, eine direkte oder indirekte Einflussnahme auf Entscheidungen zuzulassen.

${ }^{4}$ Der Öffentlichkeitsbegriff wird gegenüber dem Bürgerbegriff vorgezogen, da letzterer im engeren Wortsinn nur deutsche Staatsbürger umfasst. Bei der Öffentlichkeitsbeteiligung wird jedoch nicht zwischen Deutschen und Nichtdeutschen unterschieden. Dennoch wird im Folgenden auch auf Literatur zur Bürgerbeteiligung verwiesen, da Bürgerbeteiligung weitaus häufiger behandelt wird als Öffentlichkeitsbeteiligung und nicht immer im engeren Wortsinn verwendet wird.

${ }^{5}$ Obwohl auf den ersten beiden Stufen, der Information und des Austausches, die Öffentlichkeit keinen direkten Einfluss hat, sollen die Stufen kurz oder langfristig zu einem direkten Einfluss führen können. Beispielsweise kann die Information über ein Projekt zu einem Protest führen, obwohl dieser bei der Informationsteilung von den Entscheidern nicht eingeplant worden ist. 


\section{$4 \quad$ Öffentlichkeitsbeteiligung als Ziel für die Kommunikation von Verwaltungsbehörden}

Öffentlichkeitsbeteiligung besitzt aus rechtlicher, normativer und empirischer Perspektive eine hohe Relevanz für Verwaltungsbehörden. Rechtlich müssen Behörden eine Mindestbeteiligung gewährleisten (Müller 2010). Aus der Perspektive einer deliberativen Demokratie sollen Behörden mit Hilfe von Beteiligung die Öffentlichkeit ermächtigen, an politischen Entscheidungen teilzuhaben (Kocks und Raupp 2014, S. 278). Sie sollen das politische Engagement der Öffentlichkeit erhöhen, die Öffentlichkeit in Entscheidungsprozesse einbinden sowie vor der Öffentlichkeit Rechenschaft ablegen (Canel und Luoma-aho 2018, S. 179 ff.). Auch aus organisationaler Sicht können Behörden sich mit Hilfe von Beteiligung besser auf die Öffentlichkeit einstellen, ihre Verwaltungsleistungen optimieren, Vertrauen und Legitimität fördern sowie öffentliche Konflikte lösen (Canel und Luoma-aho 2018, S. 179 ff.). Empirische Befunde belegen ebenfalls die wichtige Bedeutung der Beteiligung für Behörden (Rademacher und Lintemeier 2015, S. 10).

Auch für die Kommunikation von Behörden nimmt Beteiligung aus rechtlicher und normativer Perspektive eine wichtige Rolle ein. Das Gesetz setzt einen Rahmen, wie Behörden mit der Hilfe von Kommunikation die Öffentlichkeit beteiligen müssen (Schulz et al. 2013, S. 19 f.). Aus normativer Sicht sollen Behörden mit Hilfe von Kommunikation die Verständigung mit der Öffentlichkeit fördern, um eine bessere Einbindung der Öffentlichkeit zu ermöglichen, sowie das Selbstbewusstsein dieser für ein Engagement stärken (Canel und Luoma-aho 2018, S. 189 f.).

Empirische Studien kommen jedoch zu widersprüchlichen Ergebnissen bezüglich der Bedeutung von Beteiligung für die Kommunikation von Behörden. Auf der einen Seite sollen laut Behörden durch Kommunikation, ,die Partizipation der Bevölkerung gefördert und der Dialog mit der Bevölkerung gepflegt werden" (Baumgartner 2010, S. 206). Auf der anderen Seite verfolgen Behördenvertreter bei der „Förderung der Transparenz über Regierungshandeln“ (Heinze 2012, S. 118) keine Beteiligungsziele im politischen Sinne. Während einige Behördenvertreter „ein dialogisches und partizipatives Verständnis von Risikokommunikation“ (Drews 2018, S. 197) offenbaren, bezeichnen andere „die Umsetzung eines solchen wechselseitigen Prozesses in der Praxis [als] sehr schwierig“ (Drews 2018, S. 197). Insgesamt legen die angeführten Studien keinen besonderen Fokus auf Beteiligung. Insbesondere die kommunale Ebene scheint, mit wenigen Ausnahmen (Glenn 2014), in diesem Feld wenig erforscht 
zu sein. Dabei ist die Kommunalebene besonders relevant für die Untersuchung von Beteiligung, da Kommunalvertreter einen besonders engen Kontakt zur Öffentlichkeit pflegen (Simmons 2014, S. 318) und der Bürgerbeteiligung einen hohen Stellenwert über die gesetzliche Mindestpflicht hinaus zuweisen (Beckmann et al. 2013, S. 22 f.). Vor diesem Hintergrund besteht Forschungsbedarf für die Untersuchung der Rolle von Beteiligung für die Kommunikation kommunaler Verwaltungsbehörden.

\section{Die Wahrnehmung von Verwaltungsbehörden bei der Öffentlichkeitsbeteiligung}

Die Vermittlung einer positiven Wahrnehmung von Behörden spielt eine wichtige Rolle bei der Kommunikation von Behörden, so bemühen sie sich um eine positive Reputation (Wæraas und Byrkjeflot 2012, S. 187). In Bezug auf Beteiligung gibt es jedoch bisher nur wenige Studien (Simmons 2014, S. 316), die aufzeigen, inwiefern die Kommunikation von Behörden das Ziel verfolgt, eine positive Wahrnehmung der Behörden bei der Beteiligung zu fördern. Damit besteht in diesem Bereich ein weiterer Forschungsbedarf.

Beteiligung kann sich sowohl positiv wie auch negativ auf die Wahrnehmung von Behörden auswirken. Laut einer Befragung gehen kommunale Behörden dabei von einer positiven Wirkung auf ihr Image aus (Beckmann et al. 2013, S. 23). Behördenvertreter auf Landes- und Kommunalebene berichten von einer vertrauensfördernden Wirkung frühzeitiger Beteiligung (Rademacher und Lintemeier 2015, S. 12). Auf der anderen Seite erscheint auch eine negative Wirkung von Beteiligung nicht unwahrscheinlich, da Ergebnisse von Beteiligungen konträr zu den Erwartungen der Öffentlichkeit ausfallen können. Dies ist darauf zurückzuführen, dass die Öffentlichkeit vielfältige Gruppen mit teilweise unterschiedlichen Interessen und Erwartungen umfasst (Pfetsch 1998, S. 235).

Behörden können demnach nur versuchen, zwischen unterschiedlichen Interessen zu vermitteln. Hierbei können sie jedoch von Teilen der Öffentlichkeit negativ wahrgenommen werden, die der Auffassung sind, dass ihre Interessen nicht gebührend einbezogen worden sind. Laut Verwaltungsbehörden nehmen mit zunehmender Konflikthaftigkeit die positiven Wirkungen von Beteiligung ab (Vetter et al. 2015, S. 296). Darüber hinaus kann Öffentlichkeitsbeteiligung Erwartungen wecken, dass Partikularinteressen erfüllt werden können, allerdings 
dürfen Verwaltungsbehörden diesen aufgrund ihrer Neutralitätsverpflichtung und Gemeinwohlorientierung nicht entgegen kommen (Schulz et al. 2013, S. 15).

Eine potenziell negative Wirkung von Beteiligung auf die Wahrnehmung führt zu Konflikten zwischen Beteiligungs- und Wahrnehmungszielen von Behörden (Fredriksson und Pallas 2016, S. 162). Die Vermutung liegt nahe, dass diese Konflikte sich auf die Kommunikation der Behörden auswirken können. Da es hierzu jedoch wenig Forschung gibt, besteht ein Bedarf ,to examine how [...] inconsistent principles of communication are translated" (Fredriksson und Pallas 2016, S. 163).

\section{Forschungsfragen}

Basierend auf den vorangegangenen Überlegungen besteht das Forschungsinteresse, Beteiligungs- und Wahrnehmungsziele sowie Konflikte zwischen diesen, bezogen auf die Kommunikation von Verwaltungsbehörden auf kommunaler Ebene, zu untersuchen. Das Forschungsinteresse lässt sich in drei Forschungsfragen übersetzen:

1. Welche Beteiligungsziele verfolgen Kommunalvertreter bei ihrer Kommunikation mit der kommunalen Öffentlichkeit?

a. Welche Informationsziele werden verfolgt?

b. Welche Austauschziele werden verfolgt?

c. Welche Einflussziele werden verfolgt?

2. Welche Wahrnehmungsziele verfolgen Kommunalvertreter bei der Beteiligung?

3. Welche Konflikte treten zwischen Beteiligungs- und Wahrnehmungszielen auf und welche Auswirkung haben diese Konflikte auf die Kommunikation?

Die Fragen richten sich allgemein an Kommunalvertreter und nicht nur an Kommunikationsverantwortliche wie Öffentlichkeitsmitarbeiter. ${ }^{6}$ Die drei Unterfragen der ersten Frage basieren auf dem oben dargelegten Verständnis politischer Öffentlichkeitsbeteiligung.

\footnotetext{
${ }^{6}$ Gemäß dem obig erläuterten Verständnis strategischer Kommunikation kommen alle Mitarbeiter als Kommunalvertreter infrage, die im Namen der Organisation mit der Öffentlichkeit kommunizieren.
} 


\section{$7 \quad$ Forschungsdesign und Vorstellung des Falls}

Um die Forschungsfragen zu beantworten, wurden für den vorliegenden Beitrag Daten analysiert, die im Rahmen eines größeren Forschungsprojektes erhoben wurden. ${ }^{7}$ Die Daten umfassen Informationen zur Kommunikation von Kommunalvertretern mit der Öffentlichkeit bei der Vorhabenplanung des Stromübertragungsnetzausbaus (nachfolgend Netzausbau). Damit ermöglichen die Daten eine Fallstudie, deren Vorteil darin besteht, bei gleichbleibendem Kontext ein Spektrum an Antworten zu den forschungsrelevanten Fragen zu erhalten (Flyvbjerg 2011, S. 301). Somit liegt der Forschungsfokus der vorliegenden Fallstudie nicht auf dem Netzausbau selbst, sondern auf der damit einhergehenden Kommunikation der Kommunalvertretern.

Der Fall: Die Kommunikation von Kommunalvertretern bei der Vorhabenplanung des Übertragungsnetzausbaus

Der aktuelle Netzausbau steht laut dem Bundesministerium für Wirtschaft und Energie im Zusammenhang mit der Energiewende, bei der Stromerzeugung dezentralisiert und verstärkt aus erneuerbaren Energien gewonnen werden soll (Bundesministerium für Wirtschaft und Energie 2017). Die Netzausbauplanung teilt sich in die Bedarfsermittlung und Vorhabenplanung auf (Bundesnetzagentur 2019). Bei der Bedarfsermittlung werden der Bedarf an Ausbau, die Anfangs- und Endpunkte von Stromnetzen sowie die bevorzugte Technologie (z. B. Überlandleitungen oder Erdkabel) festgestellt und gesetzlich verankert. Bei der Vorhabenplanung werden während der Raumordnung/Bundesfachplanung bis zu einem Kilometer breite Trassenkorridore festgelegt sowie bei der Planfeststellung konkrete Trassenverläufe beschlossen. Übertragungsnetzbetreiber (nachfolgend Netzbetreiber) agieren als Vorhabenträger und planen die Trassenverläufe. Landesbehörden bzw. die Bundesnetzagentur (nachfolgend Genehmigungsbehörden) genehmigen die Planungen. Kommunalvertreter agieren als Träger öffentlicher Belange.

Als Träger öffentlicher Belange beteiligen sich Kommunalvertreter an der Netzausbauplanung im Rahmen der gesetzlich vorgeschriebenen Öffentlichkeitsbeteiligung (Stracke 2017, S. 117). Bei der Öffentlichkeitsbeteiligung werden sie frühzeitig über die Planung von Übertragungsnetzbetreibern informiert (Stracke

\footnotetext{
${ }^{7}$ Die Daten wurden im Raumen des aus Mitteln des Landes Niedersachsen geförderten interdisziplinären Forschungsprojektes Net Future Niedersachsen an der Hochschule Osnabrück (Umansky 2017) erhoben.
} 
2017 S. 216, 241 f.). Außerdem geben sie offizielle Stellungnahmen zur Planung $\mathrm{ab}$, die von den Netzbetreibern bei der Planung und von Landesbehörden bei der Genehmigung berücksichtigt werden müssen und zu denen öffentlich Stellung genommen werden muss (Stracke 2017, S. 218 f., 262). Nach dem Ende der Planung können Kommunalvertreter gegen den Planfeststellungsbeschluss Klage einreichen (Stracke 2017, S. 268 f.).

Die formelle Rolle der Kommunalvertreter unterscheidet sich nur unwesentlich von der Rolle der breiten Öffentlichkeit. Sie haben zwar in einigen Fällen das Recht, zu einem früheren Zeitpunkt Informationen zur Planung zu erhalten und besitzen eine längere Zeit zur Abgabe formeller Stellungnahmen (Stracke 2017, S. 218 f., 262). Der weitaus wesentlichere Unterschied zur Öffentlichkeit bestehen darin, dass Kommunalvertreter oft mehr Ressourcen besitzen, um auf die formelle Netzausbauplanung einzuwirken. Abhängig von ihrer Größe beschäftigen viele kommunale Behörden Planer und Rechtsanwälte, die aus fachlicher Perspektive Stellungnahmen und Klagen wirksamer gestalten können. Außerdem besitzen viele Kommunen finanzielle Ressourcen, um fachliche Beratung in Auftrag zu geben.

Die informelle Rolle der Kommunalvertreter bei der Vorhabenplanung des Netzausbaus weist deutliche Unterschiede zur Rolle der breiten Öffentlichkeit auf. Da kommunale Behörden offiziell die Interessen der kommunalen Öffentlichkeit vertreten, werden sie von Netzbetreibern oft als Multiplikatoren (Fuhrberg und Umansky 2016, S. 121) und von Genehmigungsbehörden als lokale Anker (Fuhrberg und Umansky 2016, S. 122) wahrgenommen und bei der informellen Beteiligung bevorzugt einbezogen. Es wird von ihnen erwartet, dass sie Informationen zwischen den Betreibern bzw. Genehmigungsbehörden und der Öffentlichkeit vermitteln und Einfluss auf die Öffentlichkeit nehmen können. Gleichzeitig wissen Netzbetreiber auch, dass kommunale Behörden die Öffentlichkeit auch gegen die Planung mobilisieren und so die Arbeit der Netzbetreiber und Genehmigungsbehörden erschweren können. Aus diesen und weiteren Gründen erhalten kommunale Behörden von den Netzbetreibern mehr Einfluss auf die informelle Netzausbauplanung als die breite Öffentlichkeit. Insgesamt lässt sich feststellen, dass Kommunalvertreter aufgrund ihrer besonderen formellen und informellen Einflussmöglichkeiten auf die Netzausbauplanung, die kommunale Öffentlichkeit bei der Beteiligung an der Vorhabenplanung des Netzausbaus unterstützen können.

Die Kommunikation der Kommunalvertreter bei der Vorhabenplanung des Netzausbaus ist daher als Fall für die Erforschung von Beteiligungs- und Wahrnehmungszielen gut geeignet. Die Beteiligung bei der Planung des Netzausbaus hat für verschiedene Akteure der Öffentlichkeit einen hohen Stellenwert (Henseling 
et al. 2016, S. 30). So erachten Bürger und Bürgerorganisationen eine Beteiligung über die formelle Beteiligung hinaus und die Aufnahme ihrer Anregungen in den Planungsprozess als wichtig (Henseling et al. 2016, S. 31 f.).

Auch in der Politik gilt Beteiligung bei Infrastrukturprojekten, wie dem Netzausbau, als ein wesentlicher Faktor (Rademacher und Lintemeier 2015, S. 10). Entsprechend ist davon auszugehen, dass das Thema Beteiligung beim Netzausbau in der Kommunikation der Kommunalvertreter, die im direkten Kontakt mit der Öffentlichkeit stehen, eine wichtige Rolle spielt. Gleichzeitig trifft der Netzausbau bei vielen Bürgern und Bürgervertretern auf eine geringe Zustimmung (Rademacher und Lintemeier 2015, S. 22). Ebenso gehen verschiedene Teile der Öffentlichkeit davon aus, dass „es zu erheblichem Widerstand durch vom Netzausbau betroffene Bürger kommt" (Rademacher und Lintemeier 2015, S. 28). Die hohe Betroffenheit und Konflikthaftigkeit lässt vermuten, dass Kommunalvertreter, die die Interessen der Kommunen zu vertreten haben, u. a. mit öffentlichen Erwartungen konfrontiert werden können, auch beispielsweise mit der Forderung, Bürger bei der Ablehnung des Netzausbaus zu unterstützen. Die Erfüllung oder Enttäuschung dieser Erwartungen kann mit Konsequenzen für die Wahrnehmung der Behörden verbunden sein. Somit sollten auch Wahrnehmungsziele die Kommunikation der Behörden prägen. Der Fall des Netzausbaus erscheint außerdem interessant, da die formelle Rolle der Kommunalvertreter als Träger öffentlicher Belange großen informellen Spielraum zulässt, und die Kommunalvertreter bewusst kommunikative Entscheidungen treffen können.

\section{Forschungsvorgehen}

Die Daten, die für den vorliegenden Beitrag analysiert wurden, sind im Rahmen eines Forschungsprojektes mithilfe einer qualitativen Untersuchung erhoben worden. Der induktive Forschungsansatz ist für das vorliegende Forschungsinteresse geeignet, da die Forschungsfragen, wie oben dargestellt, bisher noch wenig erforscht sind. Die offene Datenerhebung ermöglicht, Einblicke in die vielfältigen Wahrnehmungs- und Beteiligungsziele der Kommunikation sowie über tief gehende Konflikte zwischen den Zielen zu erlangen. Die qualitative Datenerhebung umfasste leitfadengestützte Expertengespräche als eine Methode (Klemm und Liebold 2017, S. 309 f.). Diese wurde für die vorliegende Studie genutzt, da sie den Vorteil bietet, sowohl allgemeine Forschungsfragen direkt ansprechen zu können, als auch sich bei den Antworten und im Gesprächsverlauf an den jeweiligen Gesprächspartnern zu orientieren. 
Das Datenmaterial enthält 17 Expertengespräche mit 18 Kommunalvertretern. Die Samplingprinzipien, die der Auswahl der 18 Kommunalvertreter zugrunde liegen, wurden aus einer existierenden Studie übernommen (Umansky und Fuhrberg 2018, S. 5 f.). Das Sampling verlief in zwei Schritten. Zunächst wurden aus allen Netzausbaustrecken vier Strecken ausgewählt. ${ }^{8}$ Beim zweiten Schritt wurden 18 Kommunalvertreter identifiziert, deren Kommunen von der Planung der vier ausgewählten Strecken betroffen waren. ${ }^{9}$ Bei der Rekrutierung wurden Kommunalvertreter der bereits ausgewählten Behörden kontaktiert, die sich mit dem Thema Netzausbau beschäftigen und hierzu mit der Öffentlichkeit im Kontakt standen. Insgesamt arbeiteten von den 18 Kommunalvertretern zehn in einer Regierung (Bürgermeister/Landräte) und acht in einer Verwaltung (hauptsächlich Planer). Zwölf Kommunalvertreter arbeiteten auf Gemeinde/Stadt- und sechs auf Landkreisebene.

Die Gespräche mit den Kommunalvertretern dauerten zwischen 42 min und $85 \mathrm{~min}$, wobei die meisten Gespräche ca. $60 \mathrm{~min}$ dauerten. Bei einigen Gesprächen wurde das Struktur-Lege-Verfahren angewandt, um eine Reflexion der Gesprächspartner zu stimulieren. Die aufgenommenen und mit einem inhaltlichen Fokus transkribierten Gespräche wurden mithilfe der inhaltlichen Strukturierung der qualitativen Inhaltsanalyse analysiert (Mayring 2007, S. 89 f.). Alle Hauptkategorien sowie die Subkategorien der Beteiligungsziele wurden anhand der Forschungsfragen deduziert. Die Subkategorien wurden aus dem Datenmaterial induktiv abgeleitet. Die nachfolgend zur Illustrierung der Analyseergebnisse verwendeten Zitate wurden anonymisiert, um etwaige Nachteile für die Gesprächspartner zu vermeiden.

\footnotetext{
${ }^{8}$ Bei den ausgewählten Strecken konnte eine Betroffenheit der Öffentlichkeit vermutet werden, basierend auf der Berichterstattung und der Bevölkerungsdichte der anliegenden Kommunen. Außerdem variierten die Strecken in ihrer Länge, dem Planungsbeginn sowie ihrem Planungsstadium, sodass vielfältige Projekteigenschaften abgebildet werden konnten. Schließlich hatten die ausgewählten Strecken aus forschungspraktischen Gründen eine bestimmte maximale Entfernung zum Forschungsstandort.

${ }^{9}$ Zwei der 18 Kommunalvertreter meldeten sich auf eine Anfrage an eine Arbeitsgruppe von Kommunalvertretern, die sich mit dem Netzausbau beschäftigt. Die restlichen 16 Vertreter wurden anhand einer gleichmäßigen Verteilung auf alle vier Strecken ausgewählt. Des Weiteren waren ihre Behörden für Kommunen entlang der vier Strecken zuständig, die eine möglichst hohe Bevölkerungsdichte hatten, durch die eine hohe Betroffenheit vermutet werden konnte. Schließlich wurde auf Vielfalt bezüglich regierenden und verwaltenden Kommunalvertretern sowie Kommunalvertretern auf Landkreis- und Gemeinde/StadtEbene geachtet.
} 


\title{
$9 \quad$ Forschungsergebnisse
}

\subsection{Beteiligungsziele bei der Kommunikation kommunaler Behörden}

Die Auswertung der Expertengespräche ergab, dass Kommunalvertreter mit Hilfe von Kommunikation die kommunale Öffentlichkeit bei der Beteiligung an der Netzausbauplanung unterstützten. Sie verfolgten bei ihrer Kommunikation Beteiligungsziele, die sich in Informations-, Austausch- und Einflussziele aufgliederten.

\subsubsection{Informationsziele}

Die Informationsziele der Kommunalvertreter umfassten u. a. die Erläuterung sowohl technischer, energiepolitischer und wirtschaftlicher Zusammenhänge des Übertragungsnetzausbaus als auch rechtlicher Fragestellungen bezüglich des Planungsprozesses:

\begin{abstract}
„Das Hauptziel ist eigentlich Information und Erklären. Der Versuch zu erreichen, dass die Leute möglichst viel verstehen, [...] warum so eine Leitung erforderlich ist. Dazu muss man zumindest in Ansätzen auch bisschen technische Zusammenhänge verstehen. [...] Und man muss auch irgendwo versuchen deutlich zu machen, wie wird so ein Projekt eigentlich geplant und realisiert.“ (E1)
\end{abstract}

Kommunalvertreter teilten Informationen sowohl auf eigene Initiative mit, indem sie zum Beispiel Bürgerversammlungen organisierten (E3); auf Nachfrage, wenn sie Einladungen zu Bürgerveranstaltungen folgten (E10) oder persönlich kontaktiert wurden (E4); oder sie teilten gar keine Information (E5). In einigen Fällen war die Information detaillierter (E1) - in anderen Fällen informierten die Behörden die Öffentlichkeit nur indem sie einen Ansprechpartner nannten (E4). In den meisten Fällen bestand die kommunikative Herausforderung darin, komplizierte Informationen verständlich zu machen und die kommunale Öffentlichkeit zu überzeugen, Fehlinformationen zu korrigieren.

\subsubsection{Austauschziele bei der Öffentlichkeitsbeteiligung}

Neben Informationszielen verfolgten Kommunalvertreter auch Austauschziele. Hierbei vermittelten sie einen Austausch zwischen der kommunalen Öffentlichkeit und Vorhabenträgern sowie möglichen weiteren Akteuren. Durch den Austausch sollte sich die kommunale Öffentlichkeit eine umfassende Meinung zum Netzausbau bilden und die anderen Akteure bei ihrer Meinungsbildung beeinflussen: 
„Wir hatten auch gemeinsame Dreierrunden und Gespräche organisiert, wo die Vorhabenträgerin, die Verwaltung und die Bürgerinitiative mit an dem Tisch saßen. [...] So habe ich das zumindest wahrgenommen, dass wir als Verwaltung auch eine Plattform des Informationsaustausches bieten. [...] Was die Möglichkeit der Problematisierung und des Herausarbeitens angeht, glaube ich, können wir sehr gut zur Versachlichung beitragen." (E9)

Kommunalvertreter förderten den Austausch sowohl bei kleinen Treffen als auch bei größeren Bürgerversammlungen (E3). Da die kommunale Öffentlichkeit eine oft kritische Einstellung zum Netzausbau und den Netzbetreibern hatte, vermittelten viele Kommunalvertreter der Öffentlichkeit, Anfeindungen und eine Eskalation des Konflikts zu vermeiden sowie eine gemeinsame Basis durch rational-objektive Argumente zu schaffen. Einige Kommunalvertreter fassten ihre Rolle in diesem Zusammenhang als Vermittler auf (E8), was nicht bedeutet, dass sie grundsätzlich eine neutrale Position zum Netzausbau einnahmen, sondern eher den Austausch aus einer neutralen Position fördern wollten (E14).

\subsubsection{Einflussziele bei der Öffentlichkeitsbeteiligung}

Von Informations- und Austauschzielen abgesehen, gingen Kommunalvertreter auch direkten Einflusszielen nach. Hierbei verholfen sie der kommunalen Öffentlichkeit zu einer direkten Einflussnahme auf die Netzausbauplanung. Die Einflussnahme verlief über formelle und informelle Wege. Über den formellen Weg versuchten Kommunalvertreter mit Hilfe von Kommunikation, die Interessen der Öffentlichkeit zu verstehen, um sie nach einer Abwägung (E15) bei der offiziellen Stellungnahme zur Netzausbauplanung zu berücksichtigen: „Wir haben das, was Bürgerinnen und Bürger hier in der Bürgeranhörung gesagt haben, in unsere Stellungnahme mit einfließen lassen" (E11).

Andere Kommunalvertreter v. a. auf Landkreisebene organisierten einen Austausch mit der kommunalen Öffentlichkeit, um sich gemeinsam auf den Inhalt der Stellungnahme zu einigen (E12). Bei diesem Austausch vermittelten Kommunalvertreter den Bürgern, dass es um ein gemeinsames Vorgehen geht, bei dem Partikularinteressen einzelner zurückgestellt werden sollten. In beiden Fällen ging bei der formellen Einflussnahme die offizielle Stellungnahme in das Planfeststellungsverfahren ein und wurde von der Planfeststellungsbehörde bei der Entscheidung über die Genehmigung der Planung berücksichtigt.

Neben der formellen Einflussnahme beabsichtigten einige Kommunalvertreter auch eine informelle Einflussnahme der kommunalen Öffentlichkeit auf die Netzausbauplanung. Die informelle Einflussnahme teilte sich in eine konfrontative und eine kooperative auf. Bei der konfrontativen Einflussnahme nutzten 
Kommunalvertreter die Kommunikation, um die kommunale Öffentlichkeit zu mobilisieren, ihre oft kritische Position zum Netzausbau öffentlich kundzutun:

„Ich finde es gut, wenn es hier eine Bürgerinitiative gibt oder Personen, die sagen: ,Moment, wir machen auch [...] Druck, wir setzen uns ein, wir haben Interessen, die wir gegenüber dem Vorhabenträger und der Landentwicklung deutlich machen. [...] Wir müssen uns früh genug positionieren. Das habe ich unseren Bürgern und Bürgerinnen gesagt.“ (E3)

Der öffentliche Protest sollte Druck auf Netzbetreiber (E3) und Genehmigungsbehörden (E2) ausüben, um eine förderliche Entscheidung zu beeinflussen. Außerdem hatte öffentlicher Protest auch Bundestagsabgeordnete des betreffenden Wahlkreises im Fokus, um diese dazu aufzufordern, die rechtlichen Rahmenbedingungen von Planungen zu verändern (E6).

Im Gegensatz zur konfrontativen informellen Einflussnahme zielten einige Kommunalvertreter auf eine kooperative Einflussnahme ab. Hierbei führten Kommunalvertreter gemeinsam mit Vorhabenträgern und der Öffentlichkeit die Planung der Netzausbaustrecke durch. Dies geschah durch die Erlaubnis der Netzbetreiber, die das Mandat für die Planung besitzen: „Wir haben dann in der Gemeinde einen Trassenfindungsprozess angeschoben zusammen mit dem Vorhabenträger [...]. Wir wollten [...] zusammen mit dem Vorhabenträger eine möglichst konfliktarme Trasse [...] finden [...] mit Experten, Bürgern“ (E7).

Die kooperative informelle Einflussnahme ähnelte zwar dem oben beschriebenen Austausch, unterschied sich jedoch darin, dass es nicht alleine um Meinungsbildung, sondern vor allem um eine direkte Planungsbeeinflussung ging. Hierbei vermittelten Kommunalvertreter kommunikativ der kommunalen Öffentlichkeit die Wichtigkeit, eine mit dem Übertragungsnetzbetreiber gemeinsame Planung zu entwickeln.

\subsection{Wahrnehmungsziele bei der Kommunikation kommunaler Behörden}

Bei der Beteiligung bemühten sich Kommunalvertreter um eine positive Wahrnehmung. Sie wendeten drei verschiedene Strategien an:

1. Einige Kommunalvertreter vermittelten ihre Wertschätzung gegenüber der kommunalen Öffentlichkeit. Hierfür nahmen sie u. a. an Veranstaltungen teil, die von der kommunalen Öffentlichkeit als wichtig erachtetet wurden, um Respekt für ihre Anliegen zu demonstrieren: 
„Und wenn [...] man [...] eigentlich Wichtiges zu tun hat, dann muss man trotzdem hin, sonst sind die Menschen fassungslos vor Ort und sagen: Mensch, der lässt sich da nicht einmal blicken auf der wichtigsten Veranstaltung unseres Lebens [...].“ (E10)

2. Andere Kommunalvertreter förderten eine positive Wahrnehmung, indem sie ihr Bemühen vermittelten, an sie gerichtete Erwartungen zu erfüllen: „Die Menschen, die hier wohnen, die erwarten natürlich, dass wir ihre Interessen vertreten. [...] Sie erwarten, dass wir mit einem für alle Betroffenen möglichst guten Ergebnis herauskommen. [...] Dafür werden wir uns später auch rechtfertigen müssen“ (E1).

3. Daneben bemühten sich andere Kommunalvertreter um eine positive Wahrnehmung, indem sie der Öffentlichkeit vermittelten, dass sie zu einem gerechten Netzausbauplanungsprozess beitragen: „Also ich glaube, dass [...] Bürger [...] von uns erwarten, [...] darauf zu schauen, auf diesen Prozess, dass da auch eine gerechte Abwägung stattfindet" (E12).

$\mathrm{Zu}$ einem gerechten Prozess gehörten laut Kommunalvertretern allgemeingültige objektive Kriterien für die Netzausbauplanung, die für alle transparent gemacht wurden. Gleichzeitig gehörte zu einem gerechten Prozess die Einbeziehung der kommunalen Öffentlichkeit.

\subsection{Konflikte zwischen Beteiligungs- und Wahrnehmungszielen bei der Kommunikation kommunaler Behörden}

Konflikte zwischen Beteiligungs- und Wahrnehmungszielen entstanden, wenn die Beteiligung der Kommunalvertreter die Öffentlichkeit enttäuschte und sie dadurch negativ wahrgenommen wurden. Die Enttäuschung fußte auf gegensätzlichen Einstellungen zum Netzausbau.

\subsubsection{Gegensätzliche Einstellungen zum Netzausbau}

Die kommunale Öffentlichkeit ging laut vielen Kommunalvertretern bei der Netzausbauplanung von Partikularinteressen aus. Sie definierten ihre Interessen über eine individuelle Betroffenheit (E13). Wenn Netze in ihrer Umgebung ausgebaut werden sollten, lehnten sie den Netzausbau ab und forderten, den Netzausbau zu stoppen, an einen anderen Ort zu verlegen oder unter die Erde zu legen (E10). 
Demgegenüber befolgten einige Kommunalvertreter - laut eigener Aussagen übergeordnete Ziele. Sie lehnten den Netzausbau nicht ab, da er gesetzlich beschlossen ist (E15) oder sie erneuerbare Energie in der Kommune erzeugen wollten, die mithilfe der Netze transportiert werden sollte (E6). Auch eine von der Öffentlichkeit favorisierte räumliche Verlegung des Netzausbaus lehnten viele Kommunalvertreter ab, da dies zu Konflikten innerhalb der Kommune (E8) oder zwischen Kommunen führen könnte (E3). Ebenso standen einige Kommunalvertreter Erdkabeln kritisch gegenüber, da sie für die Landwirtschaft problematisch sind und durch die teure Technologie den Strompreis erhöhen könnten (E14). Unabhängig von der Einstellung zum Netzausbau argumentierten zusätzlich einige Kommunalvertreter, dass eine Verhinderung des Netzausbaus unwahrscheinlich sei und sie die Interessen ihrer Kommune eher vertreten würden, wenn sie sich konstruktiv an der Planung beteiligten (E7).

\subsubsection{Resultierende Konflikte zwischen Beteiligungs- und Wahrnehmungszielen}

Die Einstellungen der Kommunalvertreter zum Netzausbau wirkten sich auf ihre Beteiligung aus. Gegensätzliche Einstellungen zwischen Kommunalvertretern und der Öffentlichkeit führten zu einer Beteiligung, die von der Öffentlichkeit abgelehnt wurde und zu einer negativen Wahrnehmung der Kommunalvertreter führte. Dadurch kam es zu Konflikten zwischen Beteiligungs- und Wahrnehmungszielen. So erläuterte ein Kommunalvertreter (E7), dass er den Netzausbau nicht verhindern konnte und ihn auch nicht in eine Nachbarkommune verschieben wollte. Darum bemühte er sich um einen informellen Einfluss auf die Planung, um diese für seine Kommune möglichst vorteilhaft zu gestalten. Hierfür erarbeitete er gemeinsam mit der Öffentlichkeit Vorschläge für Trassenverläufe. Dafür wäre der Kommunalvertreter in der kommunalen Öffentlichkeit negativ wahrgenommen worden, da er den Netzausbau nicht verhindern oder verschieben wollte und mit der Planung de facto eine neue Betroffenheit ausgelöst hatte: „Das hat uns Kritik bei den Neubetroffenen eingebracht, denen wir dann irgendwo eine konfliktärmere Trasse am Allgemeinwohl orientiert unter Abwägung aller Belange in deren Korridor gesetzt haben“"(E7).

\subsubsection{Auswirkung von Konflikten auf die Kommunikation}

Kommunalvertreter nahmen bei Konflikten zwischen Beteiligungs- und Wahrnehmungszielen drei verschiedene Positionen ein. Bei der ersten Position behielten sie ihre Beteiligungsziele bei und lehnten kurzfristige Wahrnehmungsziele ab. Sie orientieren sich nicht an der Erfüllung kurzfristiger Erwartungen und folgten keiner populären Stimmung, sondern einer fachlich-vertretbaren, glaubwürdigen Position: 
„Ich habe hier keine Klassensprecherfunktion, dass ich jetzt irgendwelche Interessen vertrete, von denen ich selbst davon überzeugt bin, dass sie rechtlich überhaupt nicht haltbar sind, nur um sozusagen dem Volk nach dem Maul zu schauen. [...] Für Bürgermeister gibt es ja keine festgeschriebene Rolle. [...] Ich interpretier die eben nicht so sehr politisch, sondern mehr von der fachlichen Seite her. [...] Es geht da um eine Frage der eigenen Glaubwürdigkeit.“ (E11)

Bei der zweiten Position gegenüber Konflikten zwischen Beteiligungs- und Wahrnehmungszielen orientierten Kommunalvertreter ihre Beteiligungsziele an den Wahrnehmungszielen. Hierbei passten sie vor allem die formellen und informellen Einflussziele der Beteiligung an. Bei der formellen Einflussnahme richteten die Kommunalvertreter ihre fachlichen Stellungnahmen nach der öffentlichen Wahrnehmung aus:

„Es ist insofern für mich vielleicht einfacher gewesen, weil ich mit den engagierten Gegnern natürlich eine Meinung vertreten habe. Jetzt kann man mal sagen: ,Na siehste, hast es dir bequem gemacht.' Ja, möglicherweise bin ich [...] sogar auch deshalb in die Richtung gegangen." (E17)

Andere Kommunalvertreter, die die zweite Position einnahmen, passten ihre informelle Einflussnahme an die Wahrnehmungsziele an. Sie organisierten keine oder beteiligen sich nicht aktiv an informellen Planungen, damit sie sich nicht für eine entstandene Betroffenheit in der Kommune verantworten mussten. Grundsätzlich verdeutlichten sie der kommunalen Öffentlichkeit ihre geringe Verantwortung für den Netzausbau:

„Es ist sicherlich eine Herausforderung, den Bürgerinnen und Bürgern klar zu machen, dass das Ganze nicht passieren würde, wenn wir nur sagen würden: ,Wir wollen das alles nicht". [...] Und an den runden Tischen haben wir uns nicht aktiv beteiligt, [...] weil wir letztlich [...] nicht die aktive planerische Rolle besetzen wollten. Da muss man nämlich aufpassen: [...] Irgendeinem tuen Sie dann immer weh. [...] Da haben wir als Kommune uns sehr zurückgenommen und gesagt: Nein. [...] Da, in Anführungsstrichen, machen wir uns die Finger nicht dran schmutzig.“ (E9)

Kommunalvertreter, die eine dritte Position einnahmen, versuchten zwischen Beteiligungs- und Wahrnehmungszielen zu vermitteln. Sie behielten ihre Beteiligungsziele bei und bemühten sich um eine positive Wahrnehmung nicht über die Ergebnis-, sondern über die Prozessdarstellung. Sie vermittelten ihren positiven Beitrag zu einem gerechten Planungsprozess und versuchten, die negative Wahrnehmung in der kommunalen Öffentlichkeit zu mindern, indem sie darstellen, dass 
die ungewünschten Planungsergebnisse auf einem transparenten und einem auf allgemeingültigen rationalen Kriterien basierenden Prozess fußten, an dem die kommunale Öffentlichkeit teilnehmen durfte:

„Ich glaube auch selber nicht daran, dass man im Durchschnitt bei den betroffenen Menschen erreicht, dass die zufrieden sind mit dem Ergebnis. Sie werden ein Projekt in ihrer Nähe nach wie vor ablehnen. [...] Ich hoffe, dass aber wenigstens trotz Ablehnung in der Sache, sozusagen rational erkannt wird, dass wenigstens ein Bemühen dahintersteht, zu einer fairen und richtigen Lösung zu kommen [...] und gefühlt nicht diese ganz negative Einschätzung: ,Die machen ja eh mit uns, was sie wollen“."(E6)

\section{Zusammenfassung und Diskussion}

Die Forschungsergebnisse verdeutlichen die Rolle von Beteiligung für die Kommunikation kommunaler Behörden im Rahmen des Übertragungsnetzausbaus. Sie zeigen außerdem, wie Behörden sich mit Hilfe von Kommunikation um eine positive öffentliche Wahrnehmung bei der Beteiligung bemühen. Schließlich geben die Ergebnisse Einblicke, wie Beteiligungs- und Wahrnehmungsziele im Konflikt zueinanderstehen können und welche Auswirkungen es auf die Kommunikation kommunaler Behörden haben kann. Die Ergebnisse erlauben wissenschaftliche und praktische Ableitungen.

\subsection{Beteiligungs- und Wahrnehmungsziele}

Beteiligung spielte eine wichtige Rolle in der Kommunikationspraxis vieler Kommunalvertreter. Bei ihrer Kommunikation verfolgten Kommunalvertreter die folgenden Beteiligungsziele: 1) Informationsziele, 2) Austauschziele, und 3) Einflussziele.

1) Bei den Informationszielen bemühten sich Kommunalvertreter, komplexe Informationen der Öffentlichkeit verständlich zu machen. Die Verständlichkeit gilt in der Forschung als eine zentrale Herausforderung für die Kommunikation von Behörden (Ebert und Fisiak 2018). 2) Bei den Austauschzielen nahmen einige Kommunalvertreter eine vermittelnde Rolle ein und bemühten sich um einen sachlichen und kooperativen Umgang zwischen der kommunalen Öffentlichkeit und den Netzbetreibern. Dieses Ziel ist zum Teil sicherlich ihrer formellen Rolle als Träger öffentlicher Belange geschuldet. Sie sind weder in der Planungs- noch Genehmigungsverantwortung und können im Interesse ihrer 
Kommune, einen Meinungsaustausch zwischen verschiedenen Seiten fördern. Dennoch mag die Vermittlungsrolle auch sonst für Kommunalvertreter zum Alltag gehören, da sie aus normativer Perspektive der Neutralität und dem Allgemeinwohl verpflichtet sind und angesichts oft widersprüchlicher öffentlicher Interessen Interessensausgleich und Konfliktbewältigung betreiben (Pfetsch 1998, S. 235).

Die Vermittlung zwischen und innerhalb von Organisationen lässt sich als Boundary Spanning bezeichnen und gilt traditionell in der strategischen Kommunikationsforschung als eine wichtige Rolle für Kommunizierende (Springston und Leichty 1994). 3) Bei den Einflusszielen nahmen Kommunalvertreter informellen oder formellen Einfluss auf die Netzausbauplanung. Dabei nutzten sie die Kommunikation u. a. dafür, die Interessen der Öffentlichkeit zu verstehen, die Öffentlichkeit bei konfrontativem Einfluss zu mobilisieren und bei kooperativem Einfluss von einem gemeinsamen Vorgehen zu überzeugen. Diese Ergebnisse können Praktikern helfen, ihre eigenen Beteiligungsziele hinsichtlich der Kommunikation zu reflektieren. Für die Forschung lässt sich ableiten, dass ein politischer Beteiligungsbegriff (Arnstein 1969) für die Analyse von Beteiligung in der Kommunikationspraxis als geeignet erscheint.

Während Kommunalvertreter die kommunale Öffentlichkeit bei der Beteiligung unterstützten, bemühten sie sich um eine positive Wahrnehmung. Dafür vermittelten Kommunalvertreter (I) ihre Wertschätzung gegenüber der kommunalen Öffentlichkeit; (II) ihr Bemühen, die von der kommunalen Öffentlichkeit gewünschten Ergebnisse zu erreichen; sowie (III) ihren Beitrag zu einer fairen, transparenten und inklusiven Planung.

\subsection{Konflikte zwischen Beteiligungs- und Wahrnehmungszielen}

Die Beteiligung führte laut Kommunalvertretern zu einer negativen Wahrnehmung in der kommunalen Öffentlichkeit, wenn sie die Erwartungen der kommunalen Öffentlichkeit enttäuschte. Während die Öffentlichkeit laut Kommunalvertretern erwartete, mithilfe der Beteiligung Partikularinteressen bei der Planung durchzusetzen, verfolgten Kommunalvertreter aus ihrer Sicht eher gemeinschaftliche Interessen. Damit verdeutlichen die Forschungsergebnisse, dass Beteiligung anders als größtenteils dargestellt (Canel und Luoma-aho 2018, S. 185-188), auch negative Auswirkungen für Behörden haben kann (Simmons 2014, S. 313). Die Kommunalvertreter waren sich der negativen Auswirkungen bewusst und passten ihre Beteiligungs- oder Wahrnehmungsziele an. Eine Lösung 
für den Konflikt stellte für einige Kommunalvertreter die Vermittlung einer fairen, transparenten und inklusiven Planung dar. Damit versuchten sie, die negative Wahrnehmung bedingt durch das Planungsergebnis zu kompensieren. Diese Lösung entspricht der Vermittlung eines prozeduralen Gerechtigkeitsempfindens, das trotz ungewünschter Ergebnisse negative Wahrnehmungen mindern kann (Colquitt et al. 2001, S. 426 f.). Mithilfe der Befunde der Organisationsgerechtigkeitsforschung können Praktiker ihre Kommunikation bei konfliktreichen Infrastrukturprojekten reflektieren (Fuhrberg et al. 2016).

Unabhängig davon, wie Kommunalvertreter ihre Kommunikation aufgrund von Konflikten anpassen, ist entscheidend, dass sie ihre Kommunikation anpassen. Zum Beispiel versuchten einige Kommunalvertreter angesichts möglicher öffentlicher Kritik, ihre geringe Verantwortung für die Netzausbauplanung der Öffentlichkeit zu verdeutlichen. Andere Kommunalvertreter hoben bei ihrer öffentlichen Kommunikation Verfahrensgerechtigkeit hervor.

Das unterstützt die These, dass sich die strategische Kommunikation von Behörden durch eine Aushandlung widersprüchlicher Ziele konstituiert (Fredriksson und Pallas 2016). Dies bedeutet für die Praktiker, dass sich interne Widersprüche bei den Behörden, die erhebliche Kommunikationsherausforderungen bedeuten, wohl kaum reduzieren lassen (Clarke et al. 2006). Vielmehr sollten Praktiker Widersprüche kontinuierlich reflektieren, um auf der Basis eines besseren Komplexitätsverständnisses zu kommunizieren (Umansky und Fuhrberg 2018, S. 9 f.). Für die Kommunikationsforschung bedeutet dies, dass die Komplexität strategischer Kommunikation weder durch einzelne Faktoren noch durch einzeln untersuchte Faktoren, sondern erst durch die Beziehung zwischen vielfältigen Faktoren nachvollzogen werden kann.

\section{Literatur}

Alcántara, S., Bach, N., Kuhn, R., \& Ullrich, P. (2016). Demokratietheorie und Partizipationspraxis. Analyse und Anwendungspotentiale deliberativer Verfahren. Wiesbaden: Springer VS.

Arnstein, S. R. (1969). A ladder of citizen participation. Journal of the American Institute of Planners, 35(4), 216-224.

Baumgartner, S. (2010). Die Regierungskommunikation der Schweizer Kantone. Regeln, Organisation, Akteure und Instrumente im Vergleich. Wiesbaden: VS Verlag.

Beckmann, K. J., Bock, S., Landua, D., \& Reimann, B. (2013). Auf dem Weg, nicht am Ziel. Aktuelle Formen der Bürgerbeteiligung - Ergebnisse einer Kommunalbefragung des Deutschen Instituts für Urbanistik. Umwelt und Mensch Informationsdienst, 2, 21-27. 
Bogumil, J. (2008). Regierung und Verwaltung. In G. Breit, P. Massing, \& J. Bogumil (Hrsg.), Regierung und Regierungshandeln. Eine Einführung (S. 51-73). Schwalbach: Wochenschau.

Bundesministerium für Wirtschaft und Energie. (2017). Rahmenbedingungen für den Netzausbau, BMWI. https://www.bmwi.de/Redaktion/DE/Artikel/Energie/stromnetze-undnetzausbau-regulierung-rahmenbedingungen.html. Zugegriffen: 28. Febr. 2019.

Bundesnetzagentur. (2019). Netzausbau - Fünf Schritte. https://www.netzausbau. de/5schritte/de.html. Zugegriffen: 28. Febr. 2019.

Canel, M. J., \& Luoma-aho, V. (2018). Public sector communication. Closing the gaps between public organizations and citizens. Hoboken: Wiley.

Carpentier, N. (2016). Beyond the ladder of participation. An analytical toolkit for the critical analysis of participatory media processes. Javnost - The Public, 23(1), 70-88. https://doi.org/10.1080/13183222.2016.1149760.

Clarke, L., Chess, C., Holmes, R., \& O’Neill, K. M. (2006). Speaking with one voice. Risk Communication lessons from the US anthrax attacks. Journal of Contingencies and Crisis Management, 14(3), 160-169.

Colquitt, J. A., Conlon, D. E., Wesson, M. J., Porter, C. O. L. H., \& Ng, K. Y. (2001). Justice at the millennium. A meta-analytic review of 25 years of organizational justice research. Journal of Applied Psychology, 86(3), 425-445. https://doi.org/10.1037/00219010.86.3.425.

Czerwick, E. (1998). Verwaltungskommunikation. In O. Jarren, U. Sarcinelli, \& U. Saxer (Hrsg.), Politische Kommunikation in der demokratischen Gesellschaft. Ein Handbuch mit Lexikonteil (S. 489-495). Opladen: Westdeutscher Verlag.

Donges, P., \& Jarren, O. (2017). Akteure politischer Kommunikation. In P. Donges \& O. Jarren (Hrsg.), Politische Kommunikation in der Mediengesellschaft. Eine Einführung (4. Aufl., S. 111-131). Wiesbaden: Springer VS.

Drews, J. (2018). Risikokommunikation und Krisenkommunikation. Wiesbaden: Springer Fachmedien.

Ebert, H., \& Fisiak, I. (2018). Bürgerkommunikation auf Augenhöhe. Wie Behörden und öffentliche Verwaltung verständlich kommunizieren können (3. Aufl.). Wiesbaden: Springer Gabler.

Flyvbjerg, B. (2011). Case study. In N. K. Denzin \& Y. S. Lincoln (Hrsg.), The Sage handbook of qualitative research (S. 301-316). London: Sage.

Franz, T. (2013). Einführung in die Verwaltungswissenschaft. Wiesbaden: Springer.

Fredriksson, M., \& Pallas, J. (2016). Diverging principles for strategic communication in government agencies. International Journal of Strategic Communication, 10(3), 153164. https://doi.org/10.1080/1553118x.2016.1176571.

Fuhrberg, R., \& Umansky, D. (2016). Good guys vs. bad guys? Konflikte zwischen Selbst- und Fremdbild der Akteure als kommunikative Herausforderung für die Bürgerbeteiligung beim Übertragungsnetzausbau. In Bundesnetzagentur (Hrsg.), Wissenschaftsdialog 2016 (S. 116-129). Bonn: Bundesnetzagentur.

Fuhrberg, R., Thieme, M., \& Umansky, D. (2016). Das ist so ungerecht! Die Rolle der Gerechtigkeit in der Öffentlichkeitsbeteiligung beim Stromnetzausbau. prmagazin, 47(6), 48-53. 
Glenn, T. (2014). The management and administration of government communications in Canada. Canadian Public Administration, 57(1), 3-25. https://doi.org/10.1111/ capa. 12057.

Hallahan, K., Holtzhausen, D., van Ruler, B., Verčič, D., \& Sriramesh, K. (2007). Defining strategic communication. International Journal of Strategic Communication, 1(1), $3-35$.

Heinze, J. (2012). Regierungskommunikation in Deutschland. Wiesbaden: VS Verlag.

Henseling, C., Evers-Wölk, M., Oertel, B., Opielka, M., Kahlisch, C., \& Koestner, J. O. (2016). Ausbau der Stromnetze im Rahmen der Energiewende. Stakeholder Panel Report: 1. http://www.tab-beim-bundestag.de/de/pdf/publikationen/berichte/TAB-Arbeitsbericht-sp001.pdf. Zugegriffen: 5. Dez. 2018.

Holtzhausen, D., \& Zerfass, A. (2013). Strategic communication: Pillars and perspectives of an alternative paradigm. In A. Zerfaß, L. Rademacher, \& S. Wehmeier (Hrsg.), Organisationskommunikation und Public Relations. Forschungsparadigmen und neue Perspektiven (S. 73-94). Wiesbaden: Springer VS.

Klemm, M., \& Liebold, R. (2017). Qualitative Interviews in der Organisationsforschung. In S. Liebig, W. Matiaske, \& S. Rosenbohm (Hrsg.), Handbuch empirische Organisationsforschung (Bd. 64, S. 299-324). Wiesbaden: Springer Fachmedien.

Kocks, J. N., \& Raupp, J. (2014). Rechtlich-normative Rahmenbedingungen der Regierungskommunikation. Ein Thema für die Publizistik- und Kommunikationswissenschaft. Publizistik, 59(3), 269-284. https://doi.org/10.1007/s11616-014-0205-5.

Mayring, P. (2007). Qualitative Inhaltsanalyse. Grundlagen und Techniken (9. Aufl., Dr. nach Typoskr). Weinheim: Beltz.

Mead, G. H. (1934). Mind, self, society from the standpoint of a social behaviorist. Chicago: University of Chicago Press.

Müller, B. (2010). Die Öffentlichkeitsbeteiligung im Recht der Europäischen Union und ihre Einwirkungen auf das deutsche Verwaltungsrecht am Beispiel des Immissionsschutzrechts (Umweltrechtliche Studien, Bd. 43). Baden-Baden: Nomos.

Pfetsch, B. (1998). Regieren unter den Bedingungen medialer Allgegenwart. In U. Sarcinelli (Hrsg.), Politikvermittlung und Demokratie in der Mediengesellschaft. Beiträge zur politischen Kommunikationskultur (S. 233-252). Wiesbaden: VS Verlag.

Rademacher, L., \& Lintemeier, K. (2015). Smarte Partizipation?! Warum es noch kein Erfolgsmodell für Beteiligung und Dialog gibt. Wie deutsche Politiker über frühe Öffentlichkeitsbeteiligung bei Bau- und Infrastrukturprojekten denken. http://www. dialoggesellschaft.de/wp-content/uploads/Smarte_Partizipation.pdf. Zugegriffen: 28. Febr. 2019.

Raupp, J., \& Hoffjann, O. (2012). Understanding strategy in communication management. Journal of Communication Management, 16(2), 146-161. https://doi. org/10.1108/13632541211217579.

Raupp, J., \& Kocks, J. N. (2018). Regierungskommunikation und staatliche Öffentlichkeitsarbeit aus kommunikationswissenschaftlicher Perspektive. In J. Raupp, J. N. Kocks, \& K. Murphy (Hrsg.), Regierungskommunikation und staatliche Öffentlichkeitsarbeit. Implikationen des technologisch induzierten Medienwandels (S. 7-23). Wiesbaden: Springer VS.

Rowe, G., \& Frewer, L. J. (2005). A typology of public engagement mechanisms. Science, Technology and Human Values, 30(2), 251-290. https://doi. org/10.1177/0162243904271724. 
Schulz, S. E., Janda, T. C., \& Tischer, J. (2013). »Alles open« - Oder: Wie offen sind die Kommunikationsbeziehungen zwischen Staat und Gesellschaft ausgestaltet? In H. Hill (Hrsg.), Verwaltungskommunikation. Wissenschaftliche Analysen und praktische Beispiele (S. 9-30). Baden-Baden: Nomos.

Simmons, P. (2014). Challenges for communicators in future Australian local government. Procedia - Social and Behavioral Sciences, 155, 312-319. https://doi.org/10.1016/j. sbspro.2014.10.298.

Springston, J. K., \& Leichty, G. (1994). Boundary spanning activities in public relations. Journalism Quarterly, 71(3), 697-708.

Stracke, M. (2017). Öffentlichkeitsbeteiligung im Übertragungsnetzausbau. Akzeptanzförderung als gesetzgeberisches Leitbild. Baden-Baden: Nomos.

Umansky, D. (2017). Verständigung als Grundlage strategischer Risikokommunikation. Ein Fokus vom Forschungsprojekt Net Future Niedersachsen. Corporate Communications Journal, 4(2), 22-35.

Umansky, D., \& Fuhrberg, R. (2018). Improving risk communication and public participation through mutual understanding. Journal of Communication Management, 22(1), 2-13. https://doi.org/10.1108/JCOM-12-2016-0099.

Vetter, A., Geyer, S., \& Eith, U. (2015). Die wahrgenommenen Wirkungen von Bürgerbeteiligung. In Baden-Württemberg Stiftung (Hrsg.), Demokratie-Monitoring Baden-Württemberg 2013/2014. Studien zu Demokratie und Partizipation (S. 223-342). Wiesbaden: Springer VS.

Wæraas, A., \& Byrkjeflot, H. (2012). Public sector organizations and reputation management. Five Problems. International Public Management Journal, 15(2), 186-206. https://doi.org/10.1080/10967494.2012.702590.

Open Access Dieses Kapitel wird unter der Creative Commons Namensnennung 4.0 International Lizenz (http://creativecommons.org/licenses/by/4.0/deed.de) veröffentlicht, welche die Nutzung, Vervielfältigung, Bearbeitung, Verbreitung und Wiedergabe in jeglichem Medium und Format erlaubt, sofern Sie den/die ursprünglichen Autor(en) und die Quelle ordnungsgemäß nennen, einen Link zur Creative Commons Lizenz beifügen und angeben, ob Änderungen vorgenommen wurden.

Die in diesem Kapitel enthaltenen Bilder und sonstiges Drittmaterial unterliegen ebenfalls der genannten Creative Commons Lizenz, sofern sich aus der Abbildungslegende nichts anderes ergibt. Sofern das betreffende Material nicht unter der genannten Creative Commons Lizenz steht und die betreffende Handlung nicht nach gesetzlichen Vorschriften erlaubt ist, ist für die oben aufgeführten Weiterverwendungen des Materials die Einwilligung des jeweiligen Rechteinhabers einzuholen.

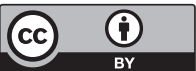

